\title{
Magnetic resonance imaging features of brain and spinal cord injury in a fatal case of isopropanol intoxication
}

This article was published in the following Dove Press journal:

International Medical Case Reports Journal

24 March 2014

Number of times this article has been viewed

\section{Parag Suresh Mahajan' Joyal Jacob Mathew ${ }^{2}$ \\ Abhilash Pulincherry \\ Jayaram' \\ Vidya Chander Negi' \\ Mohamed Milad Abu \\ Hmaira $^{2}$}

'Department of Radiology,

${ }^{2}$ Department of Medicine, Al-Khor

Hospital, Hamad Medical Corporation, Doha, Qatar

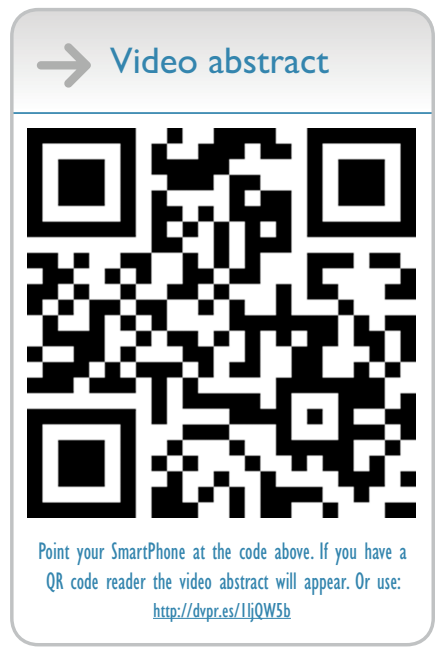

Correspondence: Parag Suresh Mahajan Department of Radiology, Al-Khor Hospital, Hamad Medical Corporation, PO Box 3050, Doha, Qatar

Tel +97444820967

Email mdfrcr@gmail.com

\begin{abstract}
A 60-year-old man presented with headache, dizziness, and disorientation one day after consumption of isopropanol along with ethanol. Computed tomography (CT) of the brain performed immediately was unremarkable. The patient collapsed within the hospital 30 minutes after the CT scan was done, and remained comatose until death, showing no improvement with symptomatic treatment. Magnetic resonance imaging of the brain and spine done 6 days after admission revealed bilaterally symmetrical hyperintensities involving the cerebral and cerebellar cortex and white matter, basal ganglia, thalami, and brainstem on T2-weighted, fluid attenuated inversion recovery and diffusion weighted images; similar hyperintensities were seen involving the swollen and edematous cervical spinal cord and cerebellar tonsillar herniation compressing the proximal cervical cord. Petechial hemorrhages were also noted within the brainstem. These features are compatible with toxic injury to the brain and cervical spinal cord. To our knowledge, the magnetic resonance imaging features of brain and spinal cord injury and cerebellar tonsillar herniation, secondary to isopropanol intoxication have not been reported in the published literature before.
\end{abstract}

Keywords: alcohol intoxication, computed tomography, isopropyl alcohol, ethyl alcohol, toxicity

\section{Introduction}

The clinical effects of toxic alcohols are mainly due to depression of both the central nervous system and myocardial function. ${ }^{1}$ There are very few published reports of the magnetic resonance imaging (MRI) features of central nervous system involvement in various types of toxic alcohol poisoning. ${ }^{1-5}$ All reports mention cerebral and/or cerebellar toxicity, also known as toxic encephalopathy. There have been no published reports of brain and spinal cord injury secondary to isopropanol (isopropyl alcohol) intoxication. ${ }^{1-5}$

\section{Case report}

A 60-year-old Caucasian merchant ship captain presented to the emergency department of our hospital with headache, dizziness, and disorientation one day after ingestion of isopropanol along with ethanol (ethyl alcohol, drinking alcohol). He had celebrated his 60th birthday aboard ship by consuming ethanol and then isopropanol, because ethanol was not available later. His colleague accompanying him to the hospital brought an empty bottle of rubbing alcohol labelled as 70\% volume per volume isopropyl alcohol with a capacity of $473 \mathrm{~mL}$ (one pint). The label on the bottle mentioned its indication for use as a first aid antiseptic and for rubbing and massaging. The patient's 
colleague mentioned that the patient had consumed most of the liquid in the bottle and that he (the colleague) had consumed a small amount. The patient's colleague did not give any recent or past history of substance abuse. The patient was disoriented and clinical examination was unremarkable. An unenhanced computed tomography (CT) scan of the brain was performed immediately and was unremarkable. He was admitted for further investigations and management. Symptomatic treatment was started.

The patient collapsed in the hospital just 30 minutes after the CT scan was done. He became deeply comatose with a score of $3 / 15$ on the Glasgow Coma Scale (a scale to

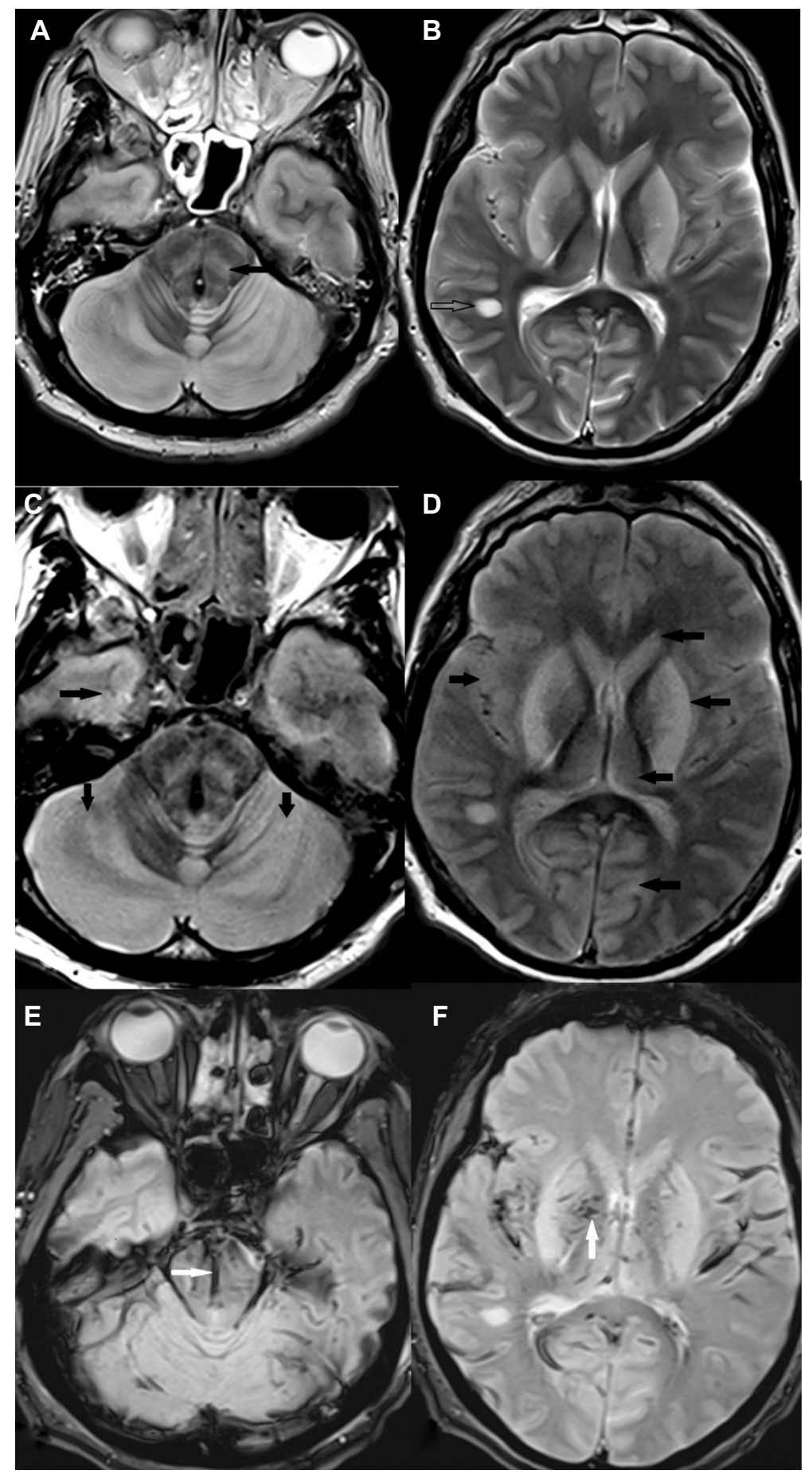

Figure I Unenhanced axial T2-weighted (A and B), fluid attenuated inversion recovery (C and $\mathbf{D})$, and T2*-weighted (E and $\mathbf{F})$ images showing hyperintensities involving the cerebral and cerebellar cortex and white matter, basal ganglia, thalami, and brainstem bilaterally (solid black arrows). A focal hyperintense lesion is noted in the right temporal periventricular white matter (open black arrow). Petechial hemorrhages are noted in the brainstem and gangliocapsular regions bilaterally (white arrows). 
assess central nervous system status where a total score of 15 indicates best and normal outcome). He also developed hypotension, with a blood pressure of $80 / 40 \mathrm{mmHg}$, a pulse rate of 65 per minute, and a respiratory rate of 8 per minute. Generalized hypotonia and absent tendon reflexes were noted on neurological examination. There was no evidence of trauma to the head or cervical spine. After administration of intravenous fluids and endotracheal intubation, the patient was admitted to the intensive care department. Blood investigations revealed leukocytosis of 26,000 (normal 4,000-10,000) white blood cells per $\mu \mathrm{L}$, a hemoglobin of 15.7 (normal 13-17) g/dL, and a mean corpuscular volume of 93 (normal 83-101) fl. The initial arterial blood gas report indicated a $\mathrm{pH}$ of 6.731 (normal 7.35-7.45), a $\mathrm{pCO}_{2}$ level of 28.2 (normal 35-48) mmHg, a pO level of 141 (normal 83-108) mmHg, a bicarbonate level of 3.5 (normal 21-28) $\mathrm{mmol} / \mathrm{L}$, and oxygen saturation of $95 \%$ (normal $95 \%-98 \%$ ). Other blood investigations revealed a blood urea nitrogen of 9.5 (normal 1.7-8.3) mmol/L, serum creatinine of 157 (normal 62-124) $\mu \mathrm{mol} / \mathrm{L}, \mathrm{Na}^{+}$of 141 (normal 134-146) $\mathrm{mmol} / \mathrm{L}, \mathrm{K}^{+}$of 6 (normal 3.5-5) $\mathrm{mmol} / \mathrm{L}, \mathrm{Cl}^{-}$of 107 (normal 96-110) $\mathrm{mmol} / \mathrm{L}, \mathrm{HCO}_{3}$ of 5 (normal 24-30) $\mathrm{mmol} / \mathrm{L}$, and capillary blood glucose of 7.2 (normal 3.3-5.5) mmol/L. Investigations were suggestive of severe metabolic acidosis and acute renal failure. His blood lactic acid level was significantly elevated at 8.7 (normal $0.5-2.2$ ) $\mathrm{mmol} / \mathrm{mL}$. Blood ketones were negative. Blood investigations were negative for the presence of ethanol. Blood levels of isopropanol and other toxic alcohols (like methanol, ethylene glycol, propylene glycol, and diethylene glycol) could not be obtained because the necessary investigations were not available. Liver enzymes, serum amylase, and lipase levels were normal.
Investigations repeated at 2-hour intervals revealed increasing renal impairment, hyperglycemia, and electrolyte imbalance (low bicarbonate levels and hyperkalemia). Follow-up arterial blood gas analysis again revealed severe acidosis. No growth was detected on cultures of urine and blood, and no crystals were found in the urine on microscopic examination. The serum pseudocholinesterase level was normal at 7,438 (normal 5,400-13,200) U/L. Calculated serum osmolarity was 310 (normal 280-301) mOsmol/L.

The patient received hemodialysis because of severe acidosis and hyperkalemia, which led to gradual improvement in his blood $\mathrm{pH}$ and lactic acid levels. He was also treated with norepinephrine (as a vasopressor) and intravenous fluids, but there was no improvement in blood pressure. He received $500 \mathrm{~mL}$ of fractionated plasma protein stat over 30 minutes, 2,000 mL of normal saline over 2 hours, and was then kept on $200 \mathrm{~mL} /$ hour of normal saline. He did not respond to intravenous fluids and within the first 2 hours was started on norepinephrine $10 \mu \mathrm{g}$ per minute, which was gradually increased up to a maximum dose of $90 \mu \mathrm{g}$ per minute. His blood pressure stabilized and he was weaned off norepinephrine after 4 days.

Unenhanced MRI scans of the brain and spine performed 6 days after hospital admission showed bilaterally symmetrical hyperintensities on T2-weighted, T1-weighted, $\mathrm{T} 2 *$-weighted, fluid attenuated inversion recovery (FLAIR), and diffusion-weighted images in the cerebral and cerebellar cortex and white matter, basal ganglia, thalami, and brainstem (Figures 1-4). A swollen and edematous cervical spinal cord was noted with T2-weighted and FLAIR hyperintensities within it (Figures 2 and 4). Cerebellar tonsillar herniation of $17 \mathrm{~mm}$ was noted to be compressing the proximal cervical cord (Figures 2 and 4). Petechial hemorrhages were noted

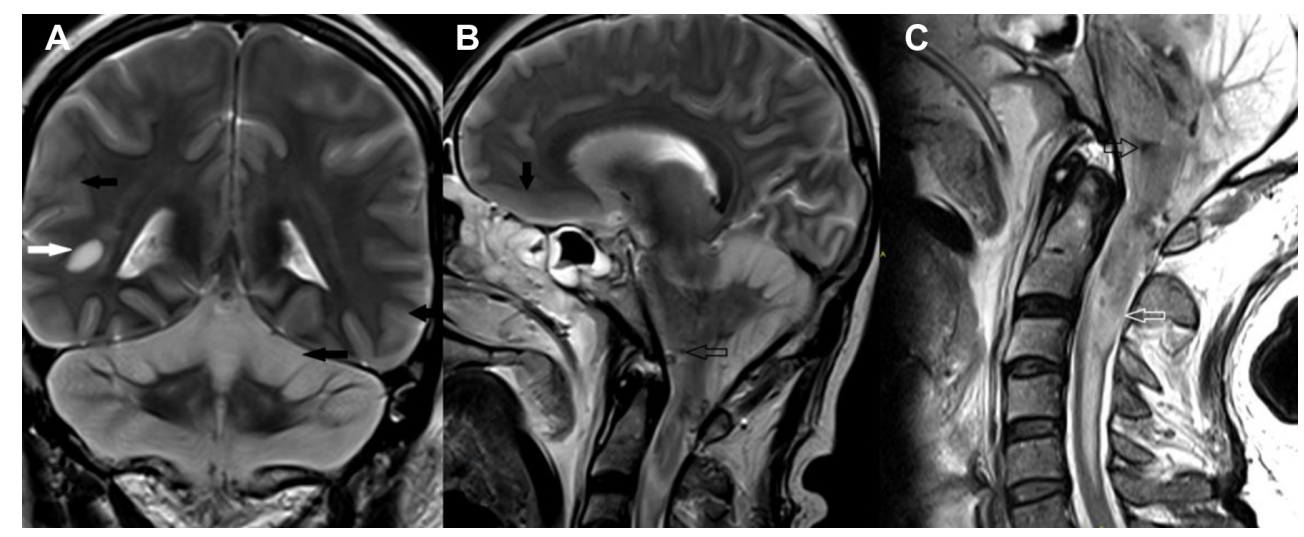

Figure 2 Unenhanced T2-weighted coronal (A) and sagittal (B) images of the brain and cervical spinal cord (C) showing hyperintensities involving the cerebellar cortex and brainstem (solid black arrows). A focal hyperintense lesion is noted in the right temporal periventricular white matter (solid white arrow). Petechial hemorrhages are noted in the brainstem (open black arrows). The cervical spinal cord is swollen and reveals hyperintense signals within it (open white arrow). 


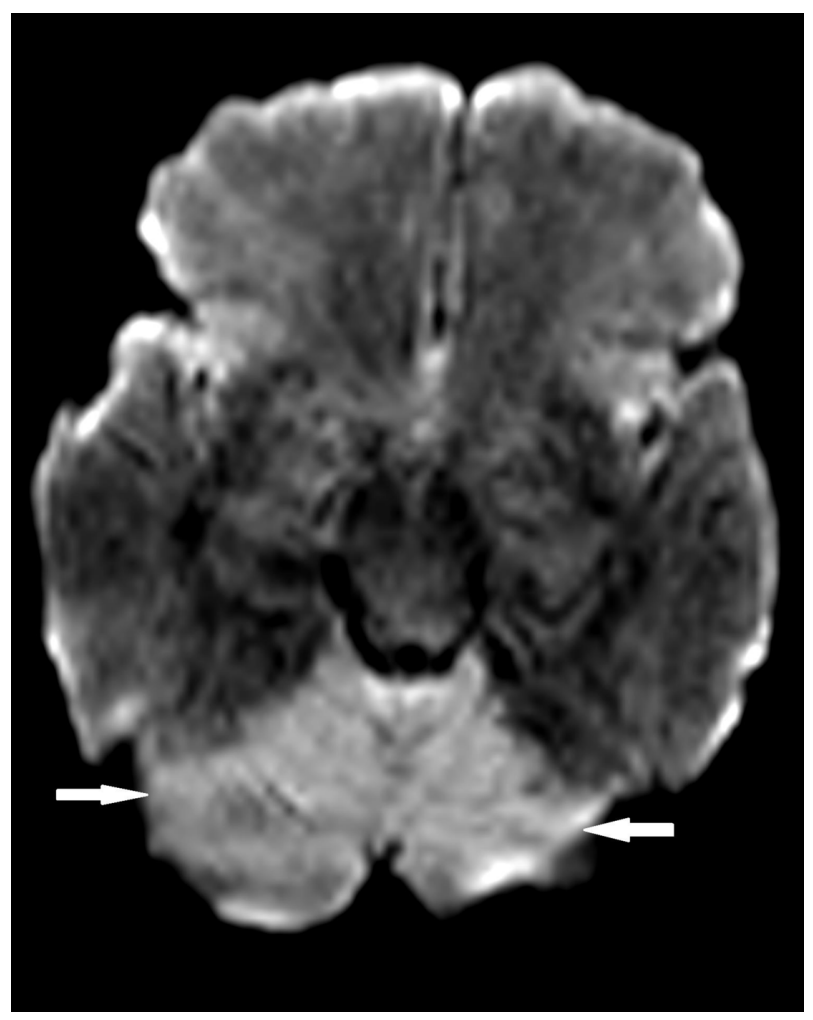

Figure 3 Axial diffusion-weighted image showing restricted diffusion involving the entire cerebellum bilaterally (arrows).

in the brainstem and the gangliocapsular regions bilaterally (Figure 1). All the features described above are compatible with toxic brain and cervical spinal cord damage. Finally, the patient expired ten days after hospital admission, despite his improving blood picture.

\section{Discussion}

Chemicals involved in alcohol intoxication are ethanol, methanol, isopropanol, ethylene glycol, diethylene glycol, and propylene glycol. Ethanol is considered as a drinking alcohol and the rest as toxic alcohols. Among the toxic alcohols, isopropanol itself is more toxic than its metabolite (acetone), while the metabolites of the rest of the toxic alcohols are more toxic than the parent alcohol. Isopropanol poisoning is characterized by an increased osmolal gap in the setting of positive serum and urine ketones and does not cause metabolic acidosis, while the rest of the toxic alcohols cause mild to severe metabolic acidosis. ${ }^{1}$ When not mixed with ethanol or other intoxicants, the signs and symptoms of intoxication may start earlier (few hours) after isopropanol ingestion and may be delayed by up to a day or more after ingestion of other toxic alcohols. Unlike methanol and ethylene glycol, isopropanol is more toxic than its metabolites; hence, alcohol dehydrogenase inhibitors are not given. ${ }^{1}$ Hemodialysis removes both isopropanol and its metabolite. ${ }^{1}$ In our case, the clinical and biochemical features were atypical for isopropanol toxicity likely due to coconsumption of ethanol and delayed patient presentation. Ingestion of approximately $200 \mathrm{~mL}$ of pure isopropanol can be lethal. ${ }^{1}$ The patient was managed symptomatically, mainly with hemodialysis. We wish to emphasize the atypical MRI features in this case that have not been reported before in cases of toxic alcohol ingestion.

In our case, MRI revealed ischemic lesions involving the brain and cervical spinal cord. These were likely due to reduced brain perfusion. ${ }^{6}$ These lesions were bilaterally symmetrical, and the distribution of the affected areas was highly indicative of a toxic injury. ${ }^{6}$ Bilateral involvement of the basal ganglia and thalami has been reported in a few cases of ethylene glycol intoxication. However, cervical spinal cord involvement and cerebellar tonsillar herniation has not been reported before in cases of toxic alcohol ingestion and even in cases of other substance abuse.

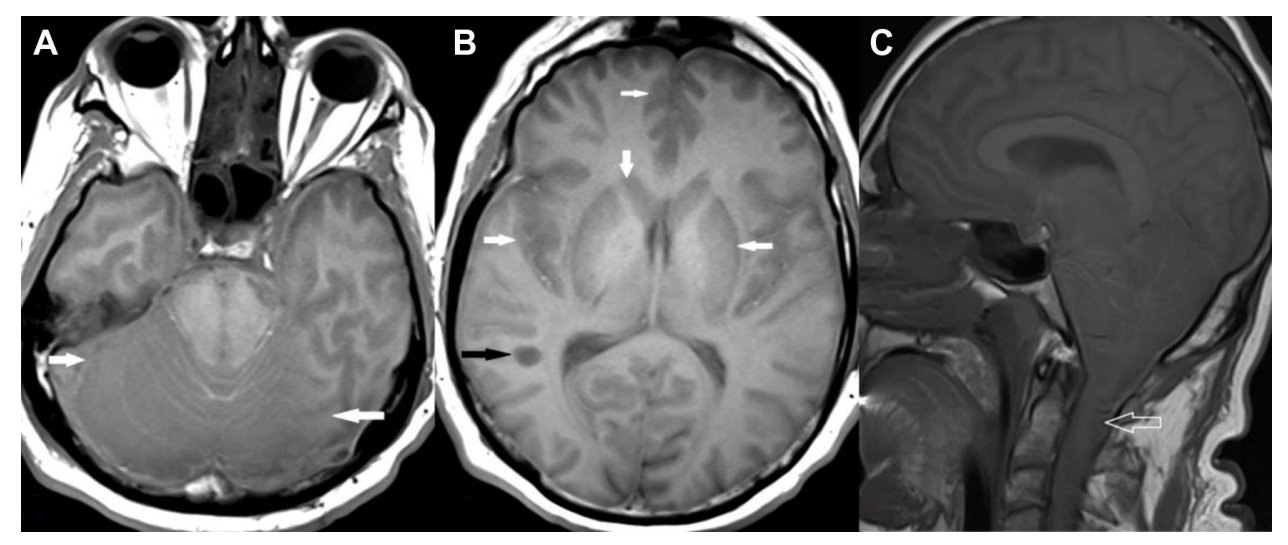

Figure 4 Unenhanced Tl-weighted axial images of brain (A and B) and sagittal image of brain and cervical spinal cord (C) showing hypointensities involving cerebral and cerebellar cortex and white matter, basal ganglia, thalami, and brainstem bilaterally (solid white arrows). A focal hypointense lesion is noted in the right temporal periventricular white matter (black arrow). The cervical spinal cord is swollen and reveals hypointense signals within it (open white arrows). 
In humans, the opioid receptors are predominantly present in the cerebellum and the limbic systems, thus causing cerebellar-predominant toxicity in cases of opioid intoxication. ${ }^{6}$ Involvement of the basal ganglia is also noticed in a few of these cases. ${ }^{6}$ A similar explanation may be given in cases of alcohol intoxication. To the best of our knowledge, this is the first reported case of cerebral, cerebellar, brainstem, and cervical spinal cord involvement on MRI secondary to isopropanol intoxication.

\section{Conclusion}

Here we present a very rare case of involvement of cerebrum, cerebellum, brainstem, and cervical spinal cord demonstrated on MRI after coconsumption of isopropanol and ethanol. To our knowledge, involvement of the cervical spinal cord and cerebellar tonsillar herniation after toxic alcohol ingestion have not been reported before in the published literature.

\section{Disclosure}

The authors report no conflicts of interest in this work.

\section{References}

1. Emadi A, Coberly L. Intoxication of a hospitalized patient with an isopropanol-based hand sanitizer. N Engl J Med. 2007;356(5):530-531.

2. Kraut JA, Kurtz I. Toxic alcohol ingestions: clinical features, diagnosis, and management. Clin J Am Soc Nephrol. 2008;3(1):208-225.

3. Jammalamadaka D, Raissi S. Ethylene glycol, methanol and isopropyl alcohol intoxication. Am J Med Sci. 2010;339(3):276-281.

4. Sharma P, Eesa M, Scott JN. Toxic and acquired metabolic encephalopathies: MRI appearance. AJR Am J Roentgenol. 2009;193(3):879-886.

5. Agarwal A, Vancil T. Toxic encephalopathy. J Gen Intern Med. 2012;27(7):876-877.

6. Corré J, Pillot J, Hilbert G. Methadone-induced toxic brain damage. Case Rep Radiol. 2013;2013:602981.

\section{Publish your work in this journal}

The International Medical Case Reports Journal is an international, peer-reviewed open-access journal publishing original case reports from all medical specialties. Previously unpublished medical posters are also accepted relating to any area of clinical or preclinical science. Submissions should not normally exceed 2,000 words or
4 published pages including figures, diagrams and references. The manuscript management system is completely online and includes a very quick and fair peer-review system, which is all easy to use. Visit http://www.dovepress.com/testimonials.php to read real quotes from published authors. 